\title{
Huaier Cream Protects against Adriamycin-Induced Nephropathy by Restoring Mitochondrial Function via PGC-1 $\alpha$ Upregulation
}

\author{
Ruochen Che, ${ }^{1}$ Chunhua Zhu, ${ }^{1,2}$ Guixia Ding, ${ }^{1,2}$ Min Zhao, ${ }^{1,2}$ Mi Bai, ${ }^{1,2}$ Zhanjun Jia, ${ }^{1,2}$ \\ Aihua Zhang, ${ }^{1,2}$ and Songming Huang ${ }^{1,2}$ \\ ${ }^{1}$ Department of Nephrology, Nanjing Children's Hospital, Nanjing Medical University, 72 Guangzhou Road, \\ Nanjing, Jiangsu 210029, China \\ ${ }^{2}$ Institute of Pediatrics, Nanjing Medical University, Nanjing, Jiangsu 210008, China
}

Correspondence should be addressed to Songming Huang; smhuang@njmu.edu.cn

Received 7 August 2014; Accepted 1 September 2014

Academic Editor: Yue Zhang

Copyright (c) 2015 Ruochen Che et al. This is an open access article distributed under the Creative Commons Attribution License, which permits unrestricted use, distribution, and reproduction in any medium, provided the original work is properly cited.

\begin{abstract}
The mechanism by which Huaier, a Chinese traditional medicine, protects podocytes remains unclear. We designed the present study to examine whether mitochondrial function restored by PGC-1 $\alpha$ serves as the major target of Huaier cream in protecting ADR nephropathy. After ADR administration, the podocytes exhibited remarkable cell injury and mitochondrial dysfunction. Additionally, ADR also reduced PGC- $1 \alpha$ both in vivo and in vitro. Following the Huaier treatment, the notable downregulation of PGC- $1 \alpha$ and its downstream molecule mitochondrial transcription factor A (TFAM) were almost entirely blocked. Correspondingly, Huaier markedly ameliorated ADR-induced podocyte injury and mitochondrial dysfunction in both rat kidneys and incubated cells as it inhibited the decrease of nephrin and podocin expression, mtDNA copy number, MMP, and ATP content. Transmission electron microscopy result also showed that Huaier protected mitochondria against ADR-induced severe mitophagy and abnormal changes of ultrastructural morphology. In conclusion, Huaier can protect podocytes against ADRinduced cytotoxicity possibly by reversing the dysfunction of mitochondria via PGC-1 $\alpha$ overexpression, which may be a novel therapeutic drug target in glomerular diseases.
\end{abstract}

\section{Introduction}

Nephrotic syndrome, characterized by massive proteinuria, hypoalbuminemia, peripheral edema, and hyperlipidemia, is one of the most common kidney diseases in children and adolescents [1]. Although glucocorticoids (GCs), the mainstay of therapy for over 50 years, are effective in most children, more than $20 \%$ develop GC-resistant nephrotic syndrome [2]. Additionally, most children who are sensitive to GCs will experience the relapse, among which 50\% will develop frequently relapsing or steroid-dependent nephrotic syndrome [3]. So combined therapy with other immunosuppressors has to be implemented. Almost all the alternative treatments we usually use today, like cyclosporine A and cyclophosphamide, have some serious adverse effects. Cyclosporine
A can induce gingival hyperplasia, hirsutism, and, the most concentrated side effects to nephrologists, nephrotoxicity [4]. The side effects of cyclophosphamide include nausea, infection, bone marrow suppression, and alopecia, of which the frequently worried about problems are possibilities of sterility and carcinoma. Given the unsatisfactory situation about nephrotic syndrome treatment, nephrologists are eager to find promising novel therapies of nephrotic syndrome. Podocyte injury plays a critical role in GC resistance in idiopathic nephrotic syndrome. Particularly in focal segmental glomerular sclerosis (FSGS), a frequent cause of end-stage renal disease with a prevalence of $4 \%$ in USA, podocytes are at significantly increased risk of injury and loss, a phenomenon called podocytopathy [5]. When podocytes are damaged, nephrotic proteinuria occurs following effacement 
of the podocyte foot processes and rearrangement of the actin cytoskeleton. This discovery has advanced the field of podocyte-targeted therapies.

Nowadays Chinese traditional herbs targeting nephropathy have drawn much attention [6-8]. Huaier cream, a Chinese traditional medicine, is a kind of Trametes robiniophila Murr., of which the extract has potent free radicalscavenging and immunomodulating properties [9]. We previously reported that Huaier exerts a protective effect in the adriamycin (ADR) nephropathy rat model by inhibiting inflammatory cytokine expression and preventing podocyte injury [10]. The proteinuria of ADR-induced rats was significantly decreased with Huaier treatments and fusion of podocyte processes was alleviated [10]. Although the protective effect of Huaier on ADR nephropathy is very impressive, the molecular mechanism remains elusive.

Growing evidences have shown the important role of mitochondrial dysfunction in mediating the podocyte injury [11-13]. Peroxisome proliferator-activated receptor- $\gamma$ coactivator $1 \alpha$ (PGC-1 $\alpha$ ) has been shown as a critical regulator of mitochondrial function in podocytes [14]. Our recent findings also demonstrated that ADR remarkably suppressed the PGC1- $\alpha$ expression in podocytes paralleled with disrupted mitochondrial function and podocytes injury. Restoration of PGC- $1 \alpha$ by adenovirus-based gene transfer markedly improved mitochondria dysfunction, abolished ROS production, and attenuated podocytes injury following ADR treatment [15]. Based on our findings described above, we speculated that Huaier may protect against podocyte injury in glomerular disease via maintaining PGC-1 $\alpha$ expression and, consequently, mitochondrial function.

Animal model of ADR nephropathy is widely used as an analogue of human FSGS showing the reduced renal function, proteinuria, podocyte dysfunction, and tubulointerstitial fibrosis [16]. The findings from ADR nephropathy model may be closely correlated to the pathogenesis and therapy of FSGS in clinic. Therefore, the striking efficacy of Huaier in treating ADR nephropathy and its underlying mechanism might be applicable to human FSGS.

\section{Materials and Methods}

2.1. Reagents. Huaier cream, the hot water extract of Trametes robiniophila Murr., was bought from Gaitianli Pharmaceutical Co. (Qidong, Jiangsu Province, China). Huaier cream is a complex of polysaccharide, proteins, and mineral substances, of which $95 \%$ is the effective substance, polysaccharide. Polysaccharide, $30,000 \mathrm{D}$, is soluble in water. The PH of aqueous solution is 5-6. Caelyx (liposomal ADR) was purchased from Merck (Whitehouse Station, NJ). Anti-cytochrome $c$ antibody and $2^{\prime}, 7^{\prime}$-dichlorofluorescein diacetate (DCFDA) were purchased from Sigma (St. Louis, MO). We used antinephrin, anti-podocin (Abcam, Cambridge, MA), anti-PGC$1 \alpha$ (Santa Cruz Biotechnology, Santa Cruz, CA), anti-COX IV, and anti- $\beta$-actin antibodies (Cell Signaling Technology, Beverly, MA). SYBR Green master mix for real-time PCR was provided by Applied Biosystems (Foster City, CA).
2.2. Animals. All experiments were performed with the approval of the experimental animal committee of Nanjing Medical University. Male Sprague-Dawley rats $(180 \pm 15 \mathrm{~g})$ were bought from Shanghai SLAC Laboratory Animals Co., Ltd. (Shanghai, China). The animals were housed and fed under standard conditions. The rats were randomly assigned to three treatment groups $(n=6$ each): the ADR group received a single, slow injection of $5 \mathrm{mg} / \mathrm{kg}$ ADR; 1 day after treatment with ADR, the Huaier-treated (Huaier + ADR) group was further treated with a daily oral gavage of $2 \mathrm{mg} / \mathrm{kg}$ Huaier cream for 15 days; the control rats received injections and oral gavages of pure saline. At the end of the experiment, rats were anesthetized with $5 \mathrm{mg} / \mathrm{kg}$ urethane. The serum was isolated and the kidneys were harvested.

2.3. Cell Culture and Treatments. MPC5 conditionally immor talized mouse podocyte clonal cells (kindly provided by Peter Mundel at Mount Sinai School of Medicine through Dr. Jie Ding at Peking University) were cultured and induced to differentiate as described [17]. Briefly, podocytes were maintained with interferon- $\gamma$ at $33^{\circ} \mathrm{C}$ for proliferation and were cultured without interferon $-\gamma$ at $37^{\circ} \mathrm{C}$ to induce differentiation. All cells were between passages 3 and 5 .

Before experiments, the cells were subcultured to $80-90 \%$ confluency in various sizes of culture vessels (six-well plates, $60 \mathrm{~mm}$ dishes), depending on the number of cells required by the protocols, and were then incubated in $1 \%$ fetal bovine serum-supplemented medium for $24 \mathrm{~h}$ to make the cells quiescent. To determine the proper stimulatory dose, we tried ADR concentrations from $50 \mathrm{nM}$ to $200 \mathrm{nM}$ and finally chose $200 \mathrm{nM}$ for use in experiments. To explore whether Huaier protects podocyte mitochondria, we pretreated cells with Huaier $(0.2 \mathrm{mg} / \mathrm{mL})$ for $1 \mathrm{~h}$ before $200 \mathrm{nM}$ ADR stimulation. The duration of stimulation was based on the protocol requirements, which are illustrated for each experiment.

2.4. Podocyte Apoptosis. After treatment, Annexin V-fluorescein isothiocyanate and propidium iodide double staining (Annexin V: fluorescein isothiocyanate apoptosis detection kit, BD Biosciences, San Diego, CA) were used to stain the podocytes following the manufacturer's protocol. We quantified apoptosis by flow cytometry (Becton, Dickinson and Company, USA).

2.5. PCR. Total RNA from cultured podocytes and renal cortex was isolated using the TRIzol total RNA isolation kit (Invitrogen, Carlsbad, CA). Single-stranded cDNAs were obtained by reverse transcription following the manufacturer's protocol. Total DNA from cultured podocytes and renal cortex was isolated using the DNeasy Tissue Kit (Invitrogen, Carlsbad, CA). The sequences of the primer pairs were in Tables 1 and 2. The mRNA and mtDNA copy numbers were detected by real-time PCR. Amplification was performed using the ABI 7300 real-time PCR detection system (Foster City, CA) with SYBR Green master mix (Applied Biosystems, Foster City, CA). Glyceraldehyde 3-phosphate dehydrogenase (GAPDH) and 18S rRNA served as internal controls of mRNA and mtDNA, respectively. The thermal 
TABLE 1: Primer sequences of rat for real-time RT-PCR.

\begin{tabular}{lcc}
\hline Gene $($ rat $)$ & Forward $\left(5^{\prime}-3^{\prime}\right)$ & Reverse $\left(3^{\prime}-5^{\prime}\right)$ \\
\hline Nephrin & ACAGCAGCCTCTTGACCAT & TGACAACCTTCAGTCCCAGT \\
Podocin & CAGCCACGGTAGTGAATGGG & TCAGGGAGGAGAGGACAAGA \\
GAPDH & CAAGTTCAACGGCACAGTCAA & TGGTGAAGACGCCAGTAGACTC \\
PGC-1 $\alpha$ & TCAGCGGTCTTAGCACTCA & TCTCTGTGGGTTTGGTGTGA \\
TFAM & AAGGTGTATGAAGCGGATTTT & CGAGGTCTTTTTGGTTTTCC \\
mtDNA & ATCCTCCCAGGATTTGGAAT & ACCGGTAGGAATTGCGATAA \\
18 rRNA & TTCGGAACTGAGGCCATGATT & TTTCGCTCTGGTCCGTCTTG \\
\hline
\end{tabular}

TABLE 2: Primer sequences of mouse for real-time RT-PCR.

\begin{tabular}{lrc}
\hline Gene $($ mouse $)$ & Forward $\left(5^{\prime}-3^{\prime}\right)$ & Reverse $\left(3^{\prime}-5^{\prime}\right)$ \\
\hline Nephrin & CCCAGGTACACAGAGCACAA & CTCACGCTCACAACCTTCAG \\
Podocin & GTGAGGAGGGCACGGAAG & AGGGAGGCGAGGACAAGA \\
GAPDH & GTCTTCACTACCATGGAGAAGG & TCATGGATGACCTTGGCCAG \\
PGC- $1 \alpha$ & CGGAAATCATATCCAACCAG & TGAGGACCGCTAGCAAGTTTG \\
mtDNA & TTTTATCTGCATCTGAGTTTAATCCTGT & CACATCATCTTACCATTTATTATCGC \\
rRNA & GGACCTGGAACTGGCAACAT & GCCCTGAACTCTTTTGTGAAG \\
\hline
\end{tabular}

cycling conditions were $95^{\circ} \mathrm{C}$ for $10 \mathrm{~min}$, followed by 40 cycles of $95^{\circ} \mathrm{C}$ for $15 \mathrm{~s}$ and $60^{\circ} \mathrm{C}$ for $1 \mathrm{~min}$.

2.6. Reactive Oxygen Species (ROS) and Mitochondrial Superoxide Measurement. DCFDA is a cell-permeable fluorogenic dye that can be oxidized by $\mathrm{H}_{2} \mathrm{O}_{2}$ to produce fluorescence and is therefore used to monitor intracellular generation of ROS as previously described [18]. To measure ROS, podocytes were cultured in six-well plates until confluence. Plates were incubated with $10 \mu \mathrm{M}$ DCFDA in the dark at $37^{\circ} \mathrm{C}$ for $30 \mathrm{~min}$. Then the podocytes were harvested and washed twice with Hanks' balanced salt solution (Sigma, St. Louis, MO), and the fluorescence was measured by flow cytometry.

The ROS generated by mitochondria were measured by MitoSOX Red reagent (Invitrogen, Carlsbad, CA), an indicator of mitochondrial superoxide. Briefly, $1 \mathrm{~mL}$ MitoSOX Red reagent working solution was incubated with podocytes adhering to the six-well plates at $37^{\circ} \mathrm{C}$ in the dark at a final concentration of $5 \mu \mathrm{M}$ for $10 \mathrm{~min}$. Then the fluorescence was measured as above.

2.7. Mitochondrial Membrane Potential (MMP). Podocyte MMP was monitored by JC-1, which is a MMP-sensitive fluorescent dye as previously described [19]. The prepared dissociated podocytes were incubated in the dark with JC$1\left(7.5 \mathrm{mM}, 30 \mathrm{~min}\right.$ at $\left.37^{\circ} \mathrm{C}\right)$. Then the cells were washed with JC-1 washing buffer precooled at $4^{\circ} \mathrm{C}$, and fluorescence was detected by flow cytometry. The relative MMP was calculated using the ratio of J-aggregate to monomer $(590 / 520 \mathrm{~nm})$. Values are expressed as the fold increase in Jaggregate/monomer fluorescence over control cells. To obtain a direct image of the fluorescence change, the podocytes were grown on glass cover slips. After treatment, the podocytes were stained by JC-1 and examined by confocal microscopy.

2.8. ATP Measurement. ATP in podocytes and renal cortex was detected with a luciferase-based bioluminescence assay kit (Sigma-Aldrich, St. Louis, MO) in a FLUOstar Optima reader following the manufacturer's instructions. Each total ATP level was calculated as luminescence normalized to protein concentration.

2.9. Western Blot. Podocytes or renal cortex homogenate (100 mg) was lysed in protein lysis buffer with protease inhibitor cocktail (Sigma, St. Louis, MO) for $20 \mathrm{~min}$ on ice. After centrifugation, the supernatant was harvested, and the lysate protein concentrations were measured by a BCA Protein Assay Kit (Pierce). Immunoblotting was performed with anti-nephrin $(1: 200)$, anti-podocin $(1: 500)$, anti-PGC$1 \alpha(1: 200)$, or anti- $\beta$-actin antibody $(1: 1000)$, followed by horseradish peroxidase-labeled secondary antibodies and ECL visualization. Bands were visualized by a GS-800 calibrated densitometer (Bio-Rad, Philadelphia, PA), and densitometry was performed by Quantity One software (Bio-Rad, Philadelphia, PA).

2.10. Measurement of Cytochrome c Release from Mitochondria to Cytoplasm. To isolate the mitochondria and cytoplasm, renal cortex was washed, homogenized, and prepared with a Mitochondria Isolation Kit for Tissue (Pierce, Rockford, IL) following the manufacturer's instructions. Western blotting was performed to detect cytochrome $c$ in mitochondria and cytoplasm. COX IV $(1: 500)$ and $\beta$-actin $(1: 1000)$ were used as the internal reference protein for mitochondria or cytoplasm, respectively.

2.11. Transmission Electron Microscopy. To evaluate mitochondrial morphology, live podocytes were collected, fixed in $1.25 \%$ glutaraldehyde/ $0.1 \mathrm{M}$ phosphate buffer, and postfixed in $1 \% \mathrm{OsO}_{4} / 0.1 \mathrm{M}$ phosphate buffer. Ultrathin sections $(60 \mathrm{~nm})$ were cut on a microtome, placed on copper grids, stained with uranyl acetate and lead citrate, and examined in an electron microscope (JEOL JEM-1010, Tokyo, Japan).

2.12. Statistical Analysis. All results are presented as mean \pm SD (standard deviation). The statistical analysis was 


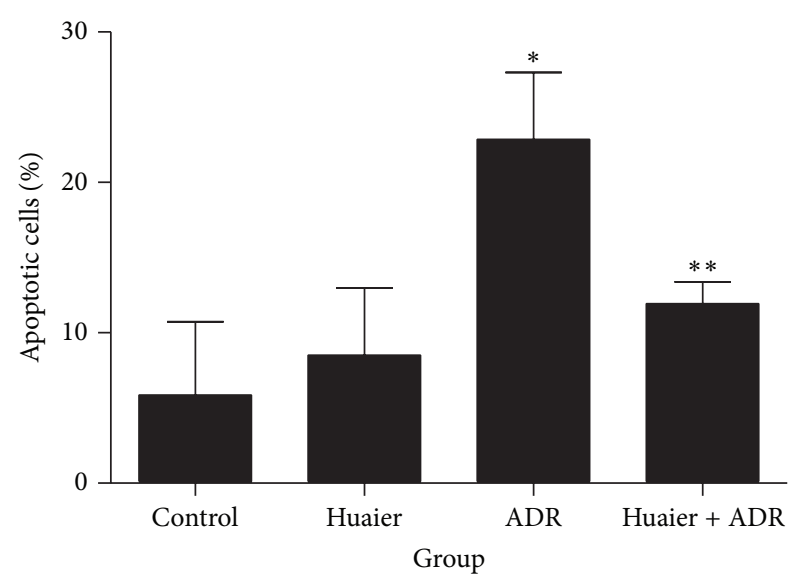

(a)

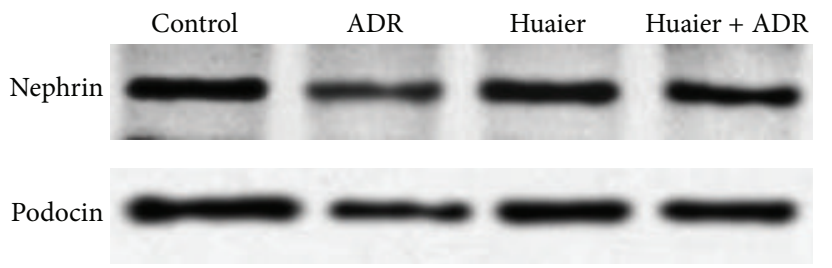

$\beta$-Actin

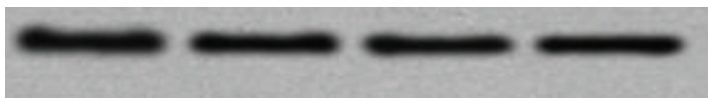

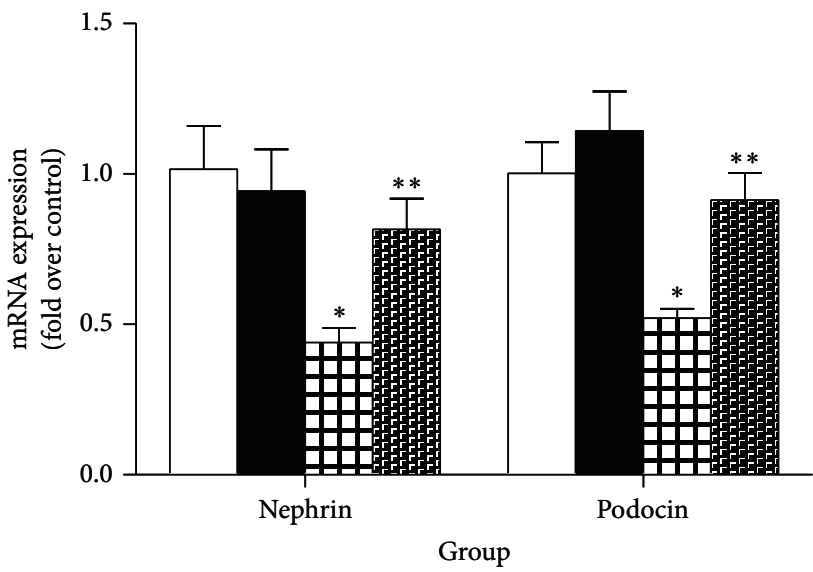

円 ADR

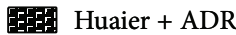

(b)

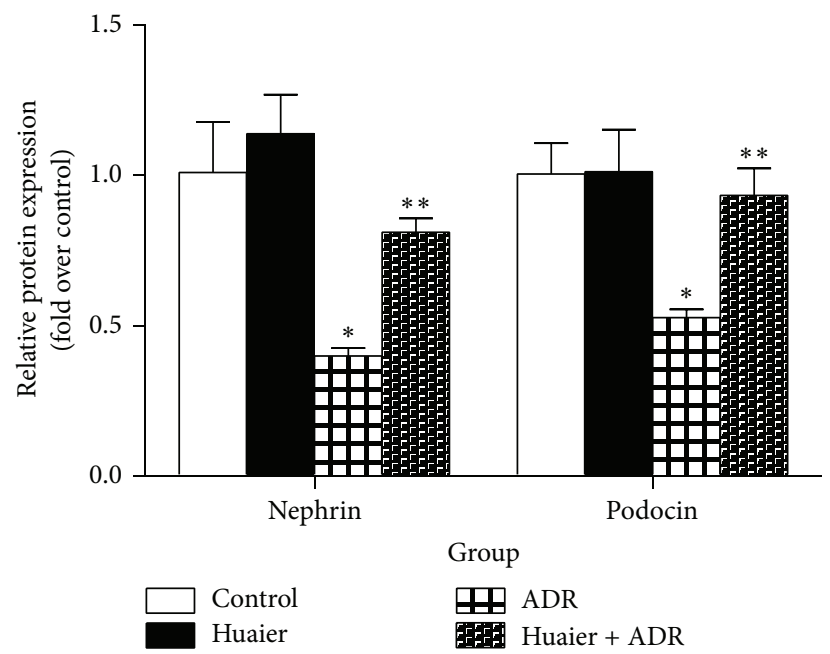

(c)

FIGURE 1: Huaier protected podocytes against ADR-induced injury in vitro. (a) Percentage of apoptotic cells. (b) Real-time RT-PCR of nephrin and podocin. (c) Western blot of nephrin and podocin. Left: representative immunoblots. Right: densitometric analysis. Podocytes were pretreated with Huaier $(0.2 \mathrm{mg} / \mathrm{mL})$ for $1 \mathrm{~h}$ followed by incubation with ADR $(200 \mathrm{nM})$ for further $48 \mathrm{~h}$ (for apoptosis analysis, (a)) or $24 \mathrm{~h}$ (for real-time RT-PCR analysis (b); immunoblotting analysis (c)). Data are expressed as the means $\pm \operatorname{SD}(n=6)$. ${ }^{*} P<0.05$ versus control. ${ }^{* *} P<0.05$ versus ADR group.

done by SPSS 16.0 using one-way analysis of variance. A $P$ value of less than 0.05 was considered statistically significant.

\section{Results}

3.1. Huaier Blocked ADR-Induced Podocyte Injury In Vitro. We investigated whether Huaier could protect podocytes against ADR-induced injury in vitro. ADR caused striking podocytes apoptosis in line with the significant downregulation of nephrin and podocin at both mRNA and protein levels (Figures $1(\mathrm{a})-1(\mathrm{c})$ ). Huaier administration remarkably decreased the percentage of apoptotic cells and strikingly alleviated the ADR-induced downregulation of nephrin and podocin (Figures 1(a)-1(c)).
3.2. Huaier Blocked ADR-Induced Mitochondrial Dysfunction In Vitro. Then we detected various parameters of mitochondrial function in vitro, including mitochondrial ROS production, mtDNA copy number, ATP content, MMP, and mitochondrial morphology. MitoSOX Red was used to measure mitochondria-derived ROS. All the mitochondrial functional parameters were disrupted by ADR as shown by reduction of mtDNA copy number and ATP content, abnormality of MMP, and overproduction of mitochondria-derived ROS. Intriguingly, such disruptions caused by ADR were robustly attenuated by Huaier (Figures 2 and 3). By transmission electron microscopy, we observed severe mitophagy and mitochondrial swelling with fracture and fusion of cristae in the ADR group. In contrast, Huaier-pretreated cells exhibited largely normal mitochondria morphology (Figure 2(a)). 

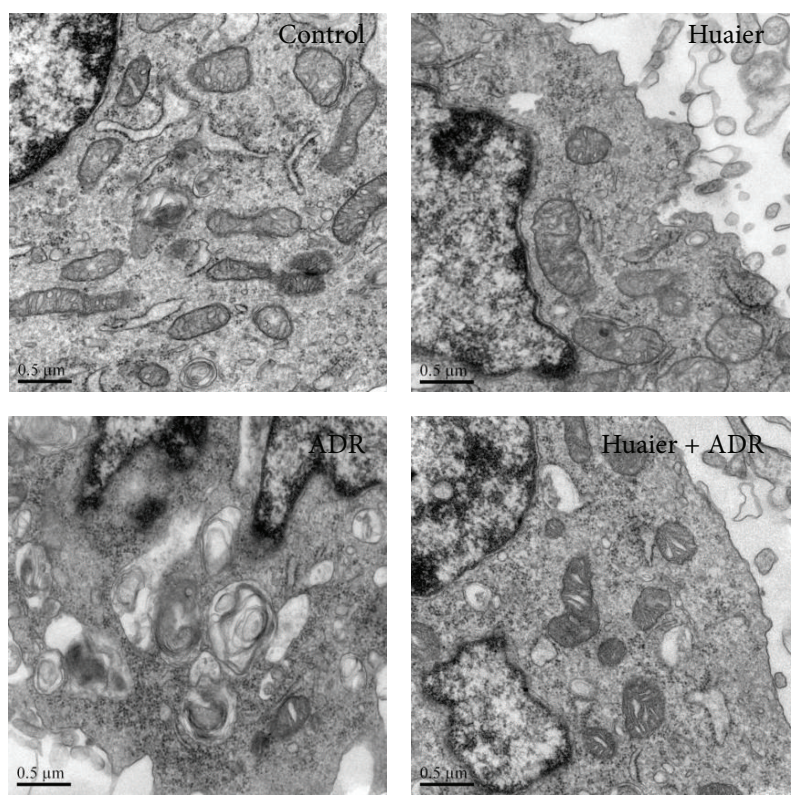

(a)

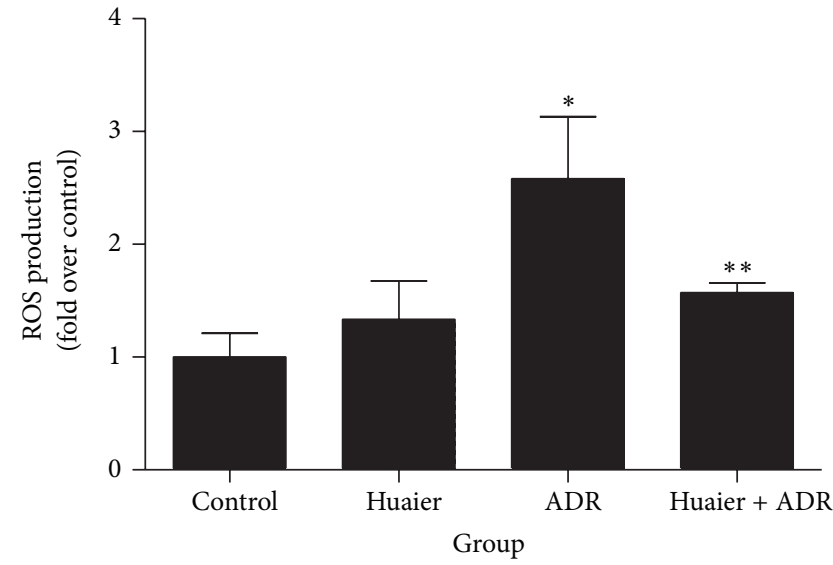

(c)
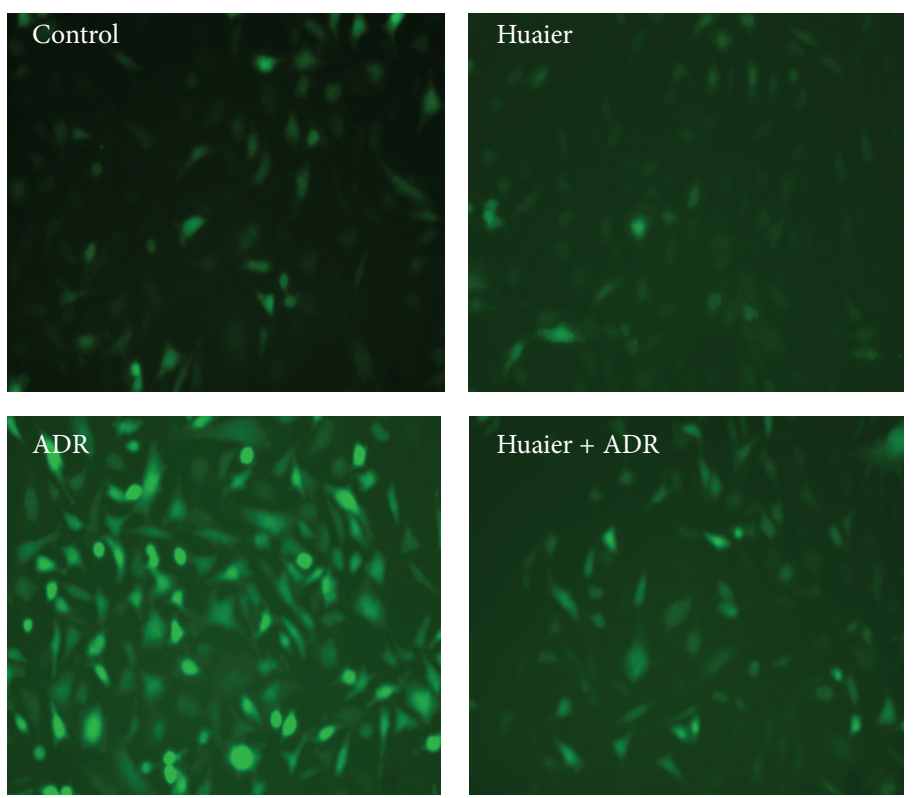

(b)

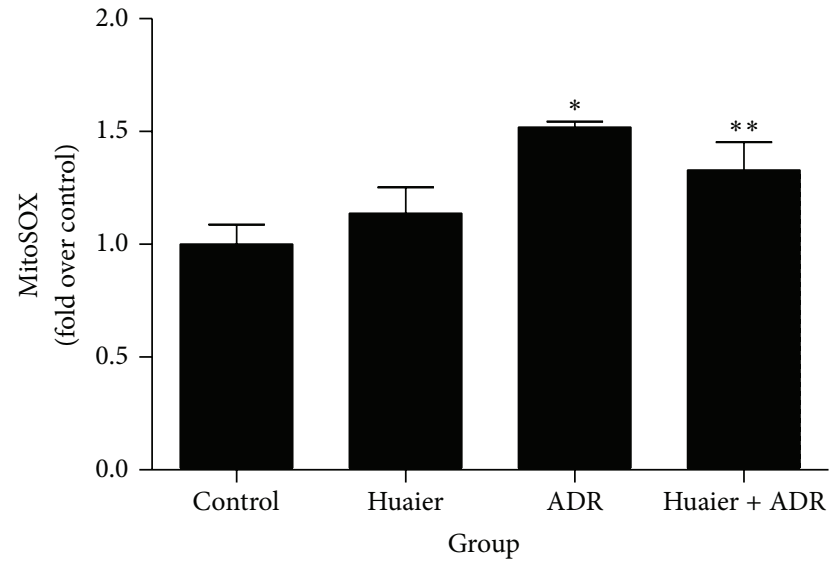

(d)

FIGURE 2: Huaier blocked ADR-induced mitochondrial dysfunction in vitro. (a) Mitochondrial morphology imaged by transmission electron microscopy. (b) Representative images of podocytes stained with dichlorodihydrofluorescein diacetate (DCFDA). (c) Quantitation of $2^{\prime}, 7^{\prime}$ dichlorofluorescein (DCF) fluorescence by flow cytometry. (d) Quantitation of MitoSOX fluorescence by flow cytometry. Podocytes were pretreated with Huaier $(0.2 \mathrm{mg} / \mathrm{mL})$ for $1 \mathrm{~h}$ followed by incubation with ADR $(200 \mathrm{nM})$ for further $24 \mathrm{~h}$. Data are expressed as the means \pm $\mathrm{SD}(n=6) .{ }^{*} P<0.05$ versus control. ${ }^{* *} P<0.05$ versus ADR group.

3.3. Huaier Ameliorated ADR-Induced Mitochondrial Dysfunction in Rat Kidneys. Our previous report had shown that Huaier significantly reduced proteinuria and prevented podocyte injury in ADR-treated rats. The kidney function was also examined. Blood urea nitrogen and serum creatinine of all the three groups were within normal range without statistical significance [10]. To investigate whether Huaier protects against ADR-indued mitochondria dysfunction in vivo, we measured ATP production, quantity of mtDNA, and cytochrome $c$ release in ADR-treated rat kidneys with or without Huaier (Figures 4(a)-4(c)). As expected, the ADR-treated group exhibited reduced ATP production and decreased mitochondrial copy number in the renal cortex, which was markedly alleviated by Huaier treatment.
Once the defense against ADR cytotoxicity fails, the MMP decreases and the mitochondrial outer-membrane pores open. Then cytochrome $c$ is released from mitochondria into the cytoplasm $[20,21]$. We observed that ADR increased the cytochrome $c$ release into the cytoplasm, which was abolished by Huaier administration, indicating a maintained normal MMP (Figure 4(c)).

3.4. Huaier Upregulated PGC-1 $\alpha$ Expression In Vivo. To further test our hypothesis, we measured PGC- $1 \alpha$ expression in ADR-treated rat kidneys (Figure 5). As shown by the results, ADR significantly reduced the PGC- $1 \alpha$ expression at both mRNA and protein levels. Meanwhile, mitochondrial transcription factor A (TFAM), a downstream molecular target 


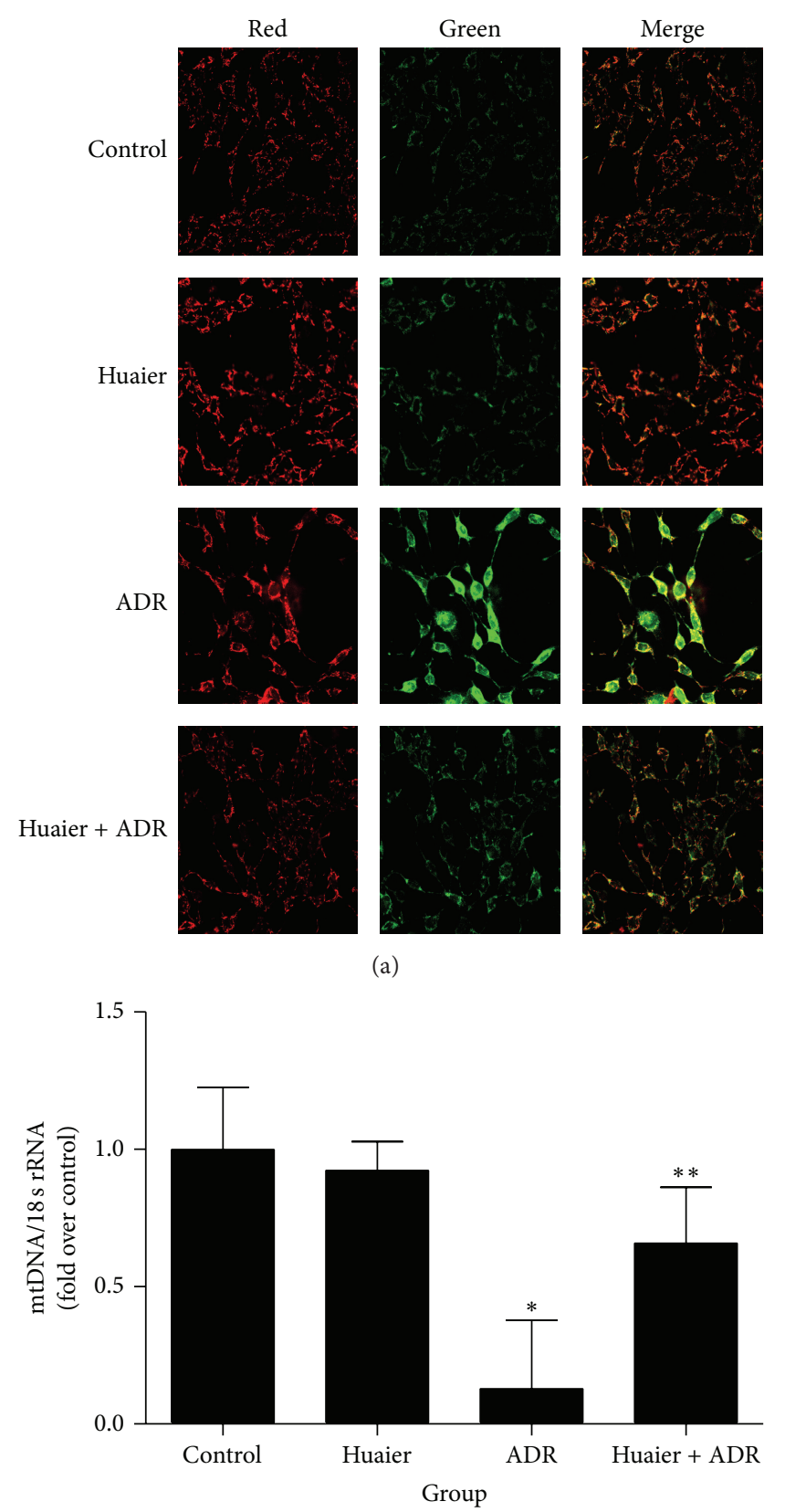

(c)

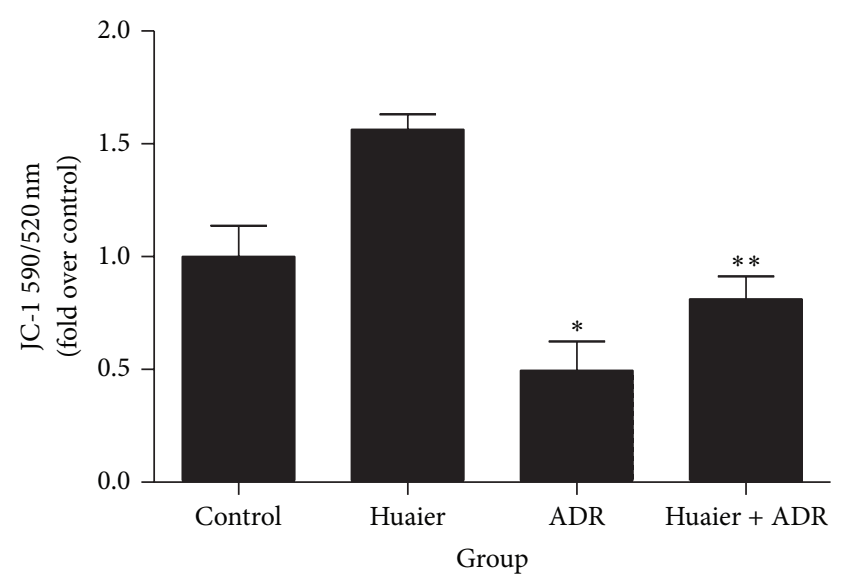

(b)

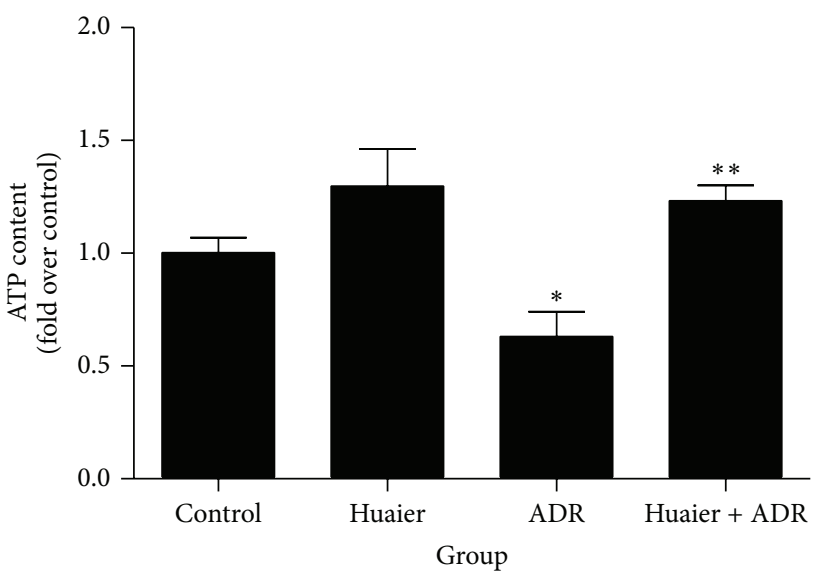

(d)

FIGURE 3: Huaier blocked ADR-induced mitochondrial dysfunction in vitro. (a) Representative images of podocytes stained with JC-1. (b) MMP measurements by flow cytometry. (c) Real-time RT-PCR analysis of mtDNA copy number. (d) ATP content. Podocytes were pretreated with Huaier $(0.2 \mathrm{mg} / \mathrm{mL})$ for $1 \mathrm{~h}$ followed by incubation with ADR $(200 \mathrm{nM})$ for further $24 \mathrm{~h}$. Data are expressed as the means \pm SD $(n=6)$. ${ }^{*} P<0.05$ versus control. ${ }^{* *} P<0.05$ versus ADR group.

of PGC- $1 \alpha$, was also dramatically decreased as determined by quantitative real-time PCR following ADR administration. Such alterations were almost completely abolished by Huaier treatment (Figure 5). These results importantly suggested that Huaier restores mitochondrial function possibly through upregulating PGC- $1 \alpha$, a critical mechanism mediating ADRinduced podocytes injury.

3.5. Huaier Upregulated PGC-1 $\alpha$ Expression In Vitro. We further examined the Huaier effect on PGC- $1 \alpha$ regulation in vitro. As shown by the data (Figure 6), ADR-induced PGC$1 \alpha$ reduction can be restored by Huaier in podocytes at both mRNA and protein levels, which indicated a direct effect of Huaier on opposing the ADR-induced PGC- $1 \alpha$ downregulation in podocytes.

\section{Discussion}

Our previous study demonstrated that ADR induced podocyte injury via disrupting PGC- $1 \alpha$ expression and 


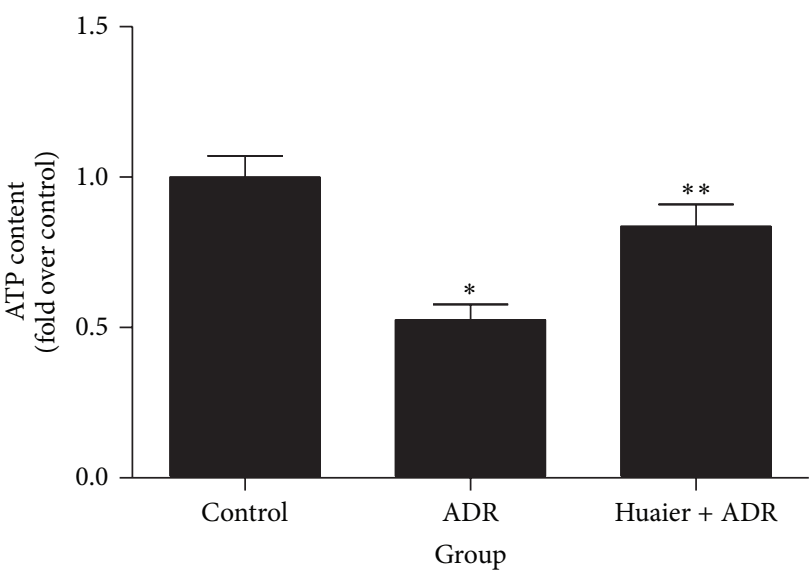

(a)

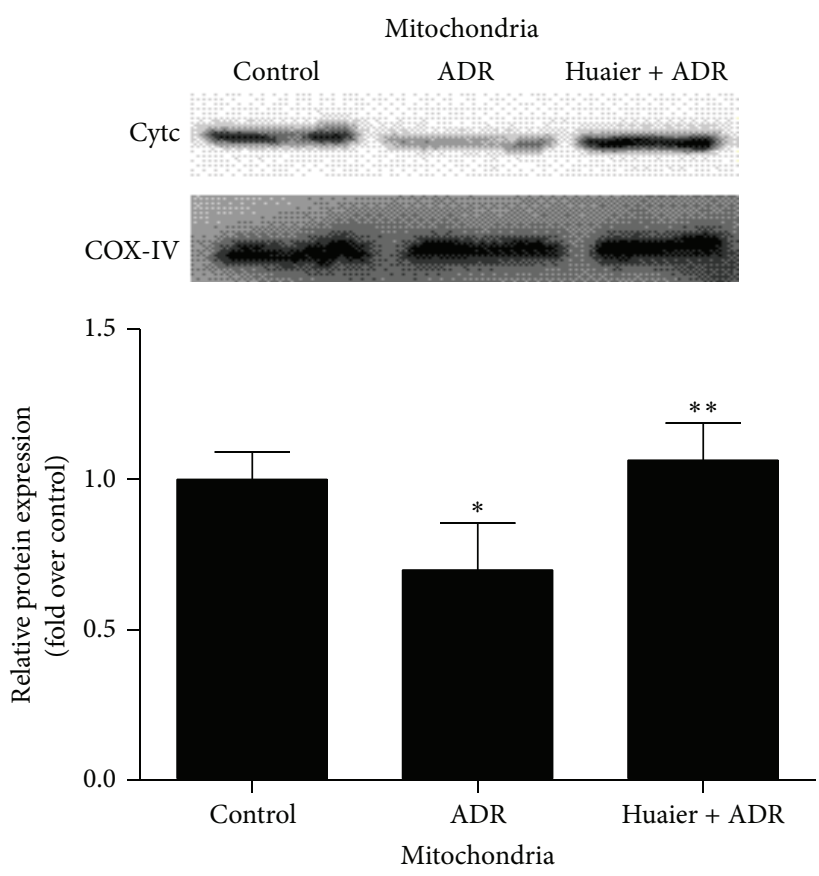

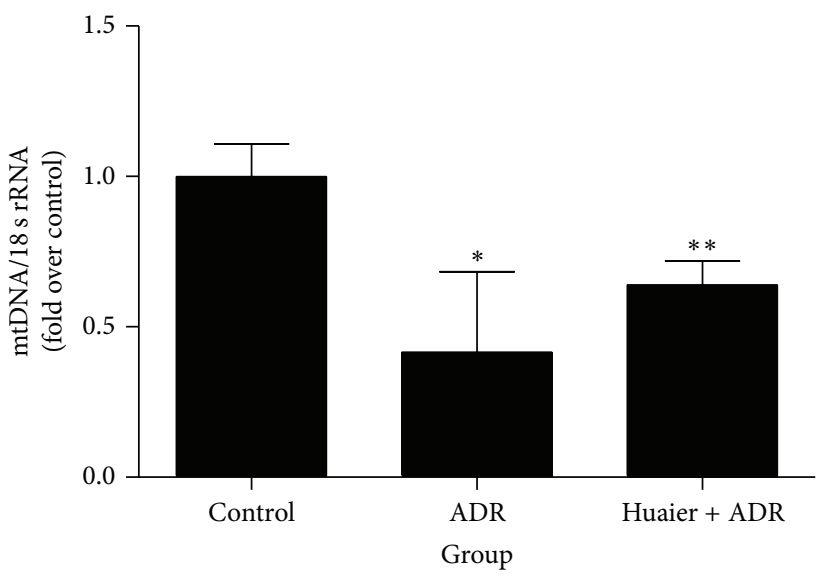

(b)

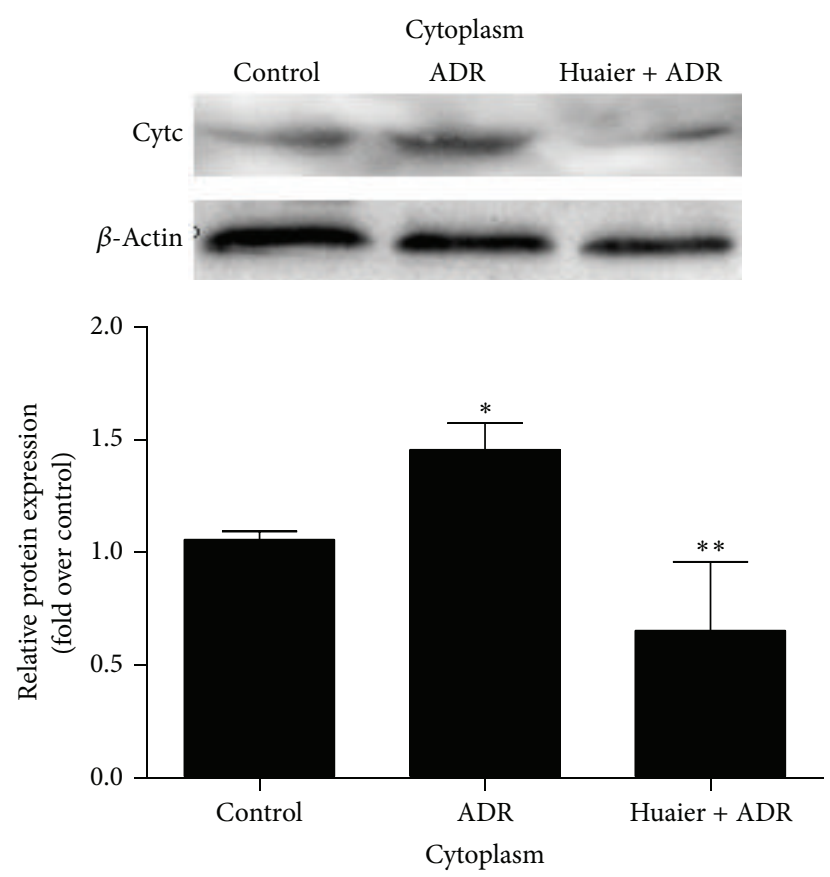

(c)

FIGURE 4: Huaier ameliorated ADR-induced mitochondrial dysfunction in rats. (a) ATP content. (b) Real-time PCR analysis of mtDNA copy number. (c) Western blot analysis of mitochondrial and cytosolic cytochrome $c$. Upper: representative immunoblots. Lower: densitometric analysis. Data are expressed as the means $\pm \operatorname{SD}(n=6) .{ }^{*} P<0.05$ versus control. ${ }^{* *} P<0.05$ versus ADR group.

mitochondrial function [15]. We also reported that Huaier cream, a traditional Chinese herb, protected against ADRinduced nephropathy in rat with unclear mechanisms. Here we presented the new finding indicating that mitochondrial function protected by PGC- $1 \alpha$ upregulation serves as the major target of Huaier cream and might be responsible for the beneficial effect of Huaier against ADR-induced podocyte injury.

Although ADR is widely used for the treatment of solid and hematogenous tumors, the high-risk side effects including ADR-related nephropathy and cardiomyopathy significantly limited its application in patients. In animal studies, ADR nephropathy is a classic model mimicking the pathology of human FSGS. ADR chiefly acts on the podocytes and interrupts the integrity of glomerular filtration barrier, which subsequently results in the proteinuria and nephrotic syndrome [16]. The podocytes injury and consequent podocytes depletion may finally result in the glomerulosclerosis [22]. Although the ADR related tissue injury received extensive studies, the mechanisms are still less understood. Lahoti et al. reported that ADR can induce mitochondrial perturbations and activate cell death genes in kidney [23]. In agreement with TS's report, we also observed that ADR caused mitochondria dysfunction and 


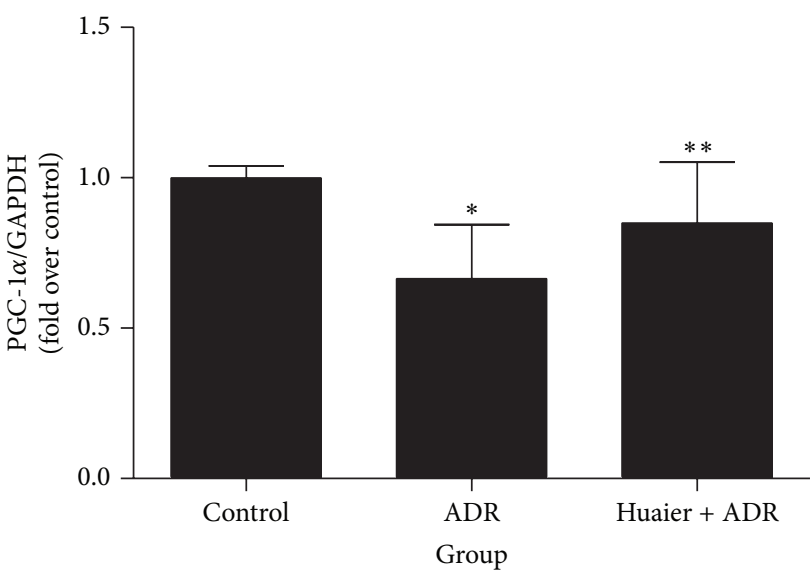

(a)

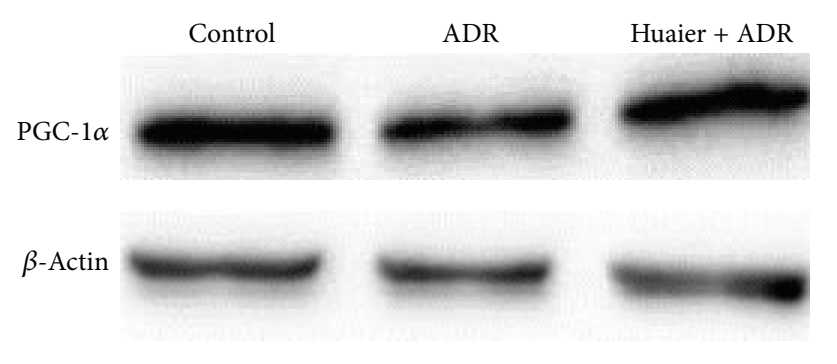

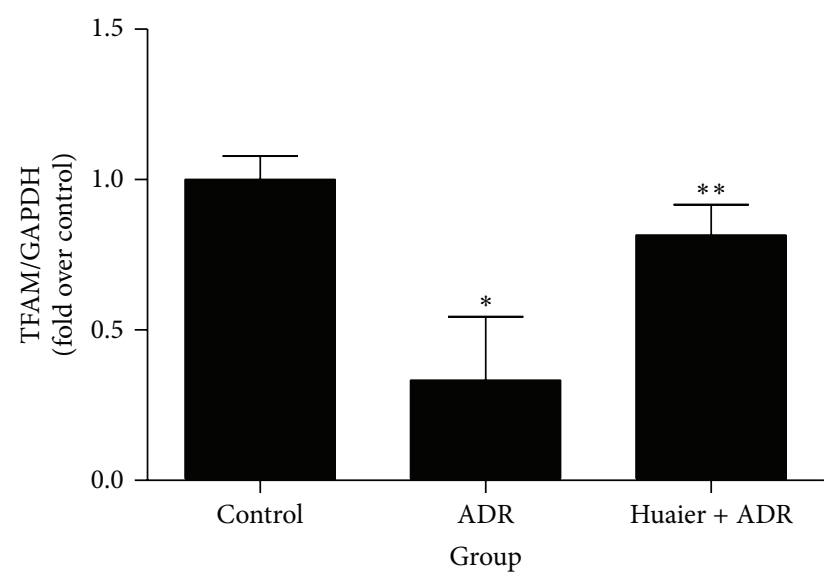

(b)

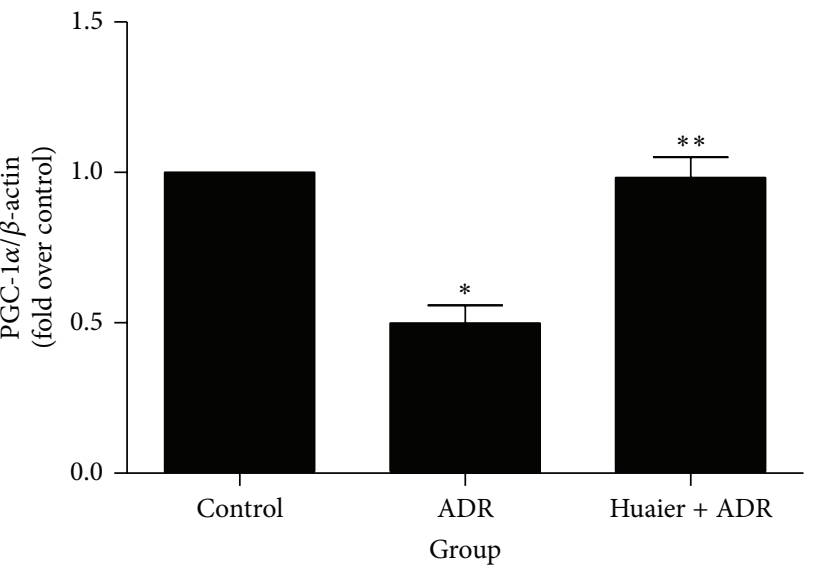

(c)

FIGURE 5: Huaier upregulated PGC-1 $\alpha$ in vivo. (a) Real-time RT-PCR of PGC-1 $\alpha$. (b) Real-time RT-PCR of TFAM. (c) Western blot of PGC$1 \alpha$. Left: representative immunoblots. Right: densitometric analysis. Data are expressed as the means $\pm \operatorname{SD}(n=6) .{ }^{*} P<0.05$ versus control. ${ }^{* *} P<0.05$ versus ADR group.

cell apoptosis in ADR-treated podocytes and kidneys [15]. And such a dysfunction of mitochondria could be responsible for ADR toxicity in kidney cells to some extent.

In the present study, Huaier treatment strikingly attenuated the podocyte injury and mitochondrial dysfunction caused by ADR in vivo and in vitro. It is well known that the injured mitochondria produce excessive intracellular ROS which further cause the damage of organelles and the decrease of ATP production due to the oxidation of mitochondrial thiols [24, 25]. The damaged mitochondria have increased permeability which allows the release of cytotoxic and proinflammatory substances, such as cytochrome $c$ and mtDNA, to start the proapoptotic cascade [20, 21]. In agreement with this theory, we found that the mitochondria disruption in ADR-treated cells or kidneys was accompanied with high levels of mitochondria-derived ROS generation and decreased ATP production. Huaier eliminated the ROS overload, restored the redox homeostasis, and protected the cells from apoptosis. Generally, mitochondria dysfunction itself initiated the mitophagy process to remove the damaged mitochondria [26]. By electron microscopy, we observed both ADR-stimulated early-stage mitophagy as shown by autophagosomes with engulfed mitochondria and late-stage mitophagy as shown by single-membrane autolysosomes with the same electron density of residual mitochondria. In the Huaier plus ADR group, Huaier restrained mitochondrial depolarization, although ADR still caused a certain degree of mitochondrial swelling and deformation.

PGC- $1 \alpha$ has an established role in maintaining the normal mitochondrial function. Convincing evidence proved that PGC- $1 \alpha$ can protect mitochondrial respiratory function and decrease mitochondrial apoptotic susceptibility [14, 27, 28]. Administration of recombinant human mitochondrial transcription factor $\mathrm{A}$, a downstream target of PGC- $1 \alpha$, protects the mitochondrial respiration in aged mice [29]. In the present study, ADR downregulated PGC- $1 \alpha$ and TFAM in both cultured cells and rat kidneys. Huaier maintained the expression of PGC- $1 \alpha$ and TFAM after ADR stimulation, suggesting a critical role of PGC- $1 \alpha$ signaling in the mechanism of Huaier action. Collectively, the findings discussed above indicated that Huaier exerted its cytoprotective effects possibly through maintaining the normal mitochondrial function 


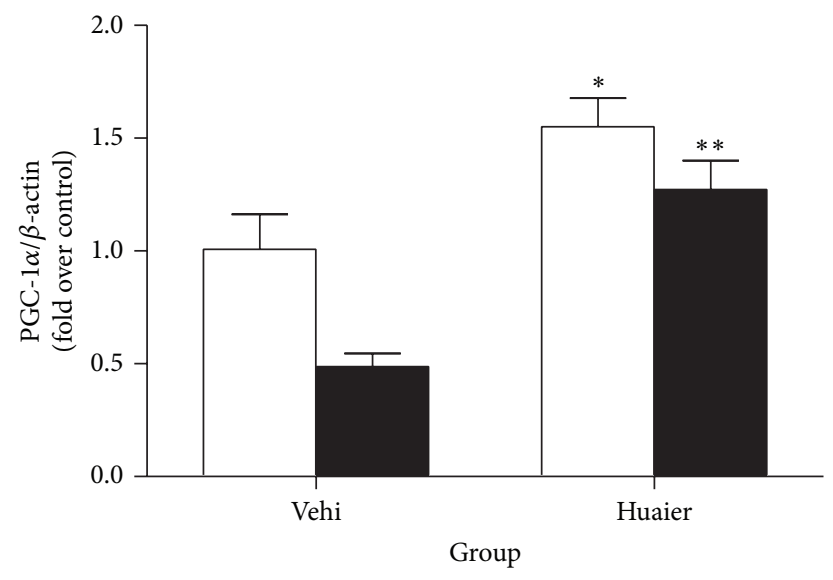

(a)
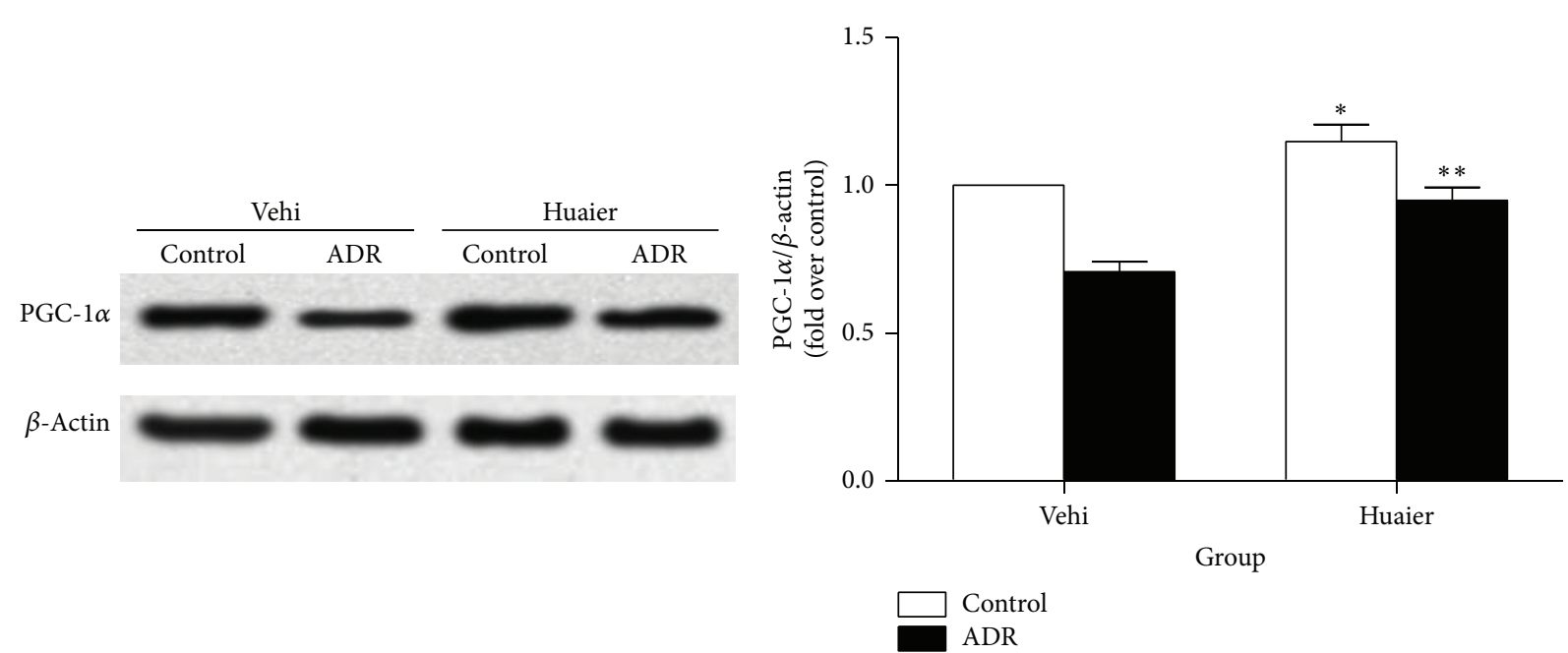

(b)

FIGURE 6: Huaier induced PGC-1 $\alpha$ expression in vitro. (a) Real-time RT-PCR of PGC-1 $\alpha$. (b) Western blot of PGC-1 $\alpha$. Left: representative immunoblots. Right: densitometric analysis. Podocytes were pretreated with Huaier $(0.2 \mathrm{mg} / \mathrm{mL})$ for $1 \mathrm{~h}$ followed by incubation with ADR $(200 \mathrm{nM})$ for further $24 \mathrm{~h}$. Data are expressed as the means $\pm \mathrm{SD}(n=6) .{ }^{*} P<0.05$ control of Huaier group versus control of vehicle group. ${ }^{* *} P<0.05$ versus ADR of Huaier group versus ADR of vehicle group.

via PGC-1 $\alpha$ expression. As the coregulator, PGC- $1 \alpha$ regulates a large number of transcription factors, like PPAR $\alpha, \operatorname{PPAR} \gamma$, estrogen receptor-related $\alpha$, FoxO1, hepatocyte nuclear factor $4 \alpha$, and nuclear respiratory factor 1 [30]. Our previous studies have shown that aldosterone-induced podocyte damage can be blocked by PPAR $\gamma$ - PGC-1 $\alpha$ dependent pathway [14, 31]. So further studies are required to investigate the mechanisms of Huaier in restoring PGC- $1 \alpha$ expression and PGC- $1 \alpha$ in regulating mitochondrial function.

Several groups reported some interesting mitochondriatargeted molecular pathways in podocytes including sirtunlPGC-1 $\alpha$ axis [14, 32], Rho-associated, coiled-coil-containing protein kinase 1 [12], and mTOR. But none of them have been applied in clinical practice. Clinically, other therapies targeting oxidative stress attenuation in podocytes include using the radical scavenger edaravone [33] and administering the antioxidants probucol and vitamin E [34]. Except for vitamin $\mathrm{E}$ [35], the other two agents are still in the animalexperiment phase $[33,34]$. Compared with these novel therapies, Huaier has irreplaceable advantages by virtue of its 1,600-year history of safe and effective clinical use [36]. In the past, Huaier was often administered to patients who are easy to get infection. Recently it has been recognized clinically that Huaier also helps to ease the proteinuria, especially for children with minimal change disease. To date, no obvious side effect was observed or reported. Based on our studies, Huaier could be a promising podocyte-targeted therapy. We are investigating the underlying pharmacological mechanisms of Huaier to more rigorously assess it for clinical therapy. Additionally, our center has joined in a doublecenter, prospective, randomized, and double-blinded clinical trial of Huaier with Children's Hospital of Fudan University 
in Shanghai. The clinical efficacy and safety of Huaier will be further evaluated.

Intriguingly, Huaier has also been applied in antitumor treatments as a complementary therapy. Wang et al. demonstrated that Huaier extract dose-dependently arrests the cell cycle, promotes apoptosis, and decreases the levels of signaling molecules, such as phosphorylated extracellular signal-regulated kinase and c-Jun $\mathrm{N}$-terminal kinase. This effect is significant at a dose greater than $2 \mathrm{mg} / \mathrm{mL}$ in vitro and $2.5 \mathrm{~g} / \mathrm{kg}$ in vivo [36]. Zhang et al. reported that a high dose of Huaier $(4-8 \mathrm{mg} / \mathrm{mL}$ ) decreases MMP and consequently induces apoptosis in a breast cancer cell line [37]. These results do not conflict with our data because the dose of Huaier we administered was much lower $(0.2 \mathrm{mg} / \mathrm{mL}$ in vitro and $2 \mathrm{mg} / \mathrm{kg}$ in vivo). To our knowledge, this is the first report that different doses of Huaier have distinct pharmacological effects. Relatively lower doses of Huaier tend to protect mitochondria, while high doses induce mitochondrial damage in cancer cells. The underlying mechanism is still unclear, waiting to be discovered.

In conclusion, our investigation indicates that Huaier protects podocytes against ADR-induced cytotoxicity (which mimics the "podocytopathies" of FSGS) by reversing mitochondrial dysfunction, most likely by maintaining the expression of PGC- $1 \alpha$ and its downstream molecules. Huaier might be a promising alternative therapy for nephrotic syndrome.

\section{Conflict of Interests}

All authors declared no competing interests, including relevant financial interests, activities, relationships, and affiliations.

\section{Acknowledgments}

This work was supported by grants from the National Basic Research Program of China 973 Program (nos. 2012CB517602 and 2013CB530604), the National Natural Science Foundation of China (nos. 81270797, 81270785, and 81325004), the Natural Science Foundation of Jiangsu Province (no. BK2012001), and the Program for New Century Excellent Talents in University (no. NCET-12-0738).

\section{References}

[1] L. A. Greenbaum, R. Benndorf, and W. E. Smoyer, "Childhood nephrotic syndrome-current and future therapies," Nature Reviews Nephrology, vol. 8, no. 8, pp. 445-458, 2012.

[2] V. D. D’Agati, "The spectrum of focal segmental glomerulosclerosis: new insights," Current Opinion in Nephrology and Hypertension, vol. 17, no. 3, pp. 271-281, 2008.

[3] S. Fujinaga, A. Endo, Y. Ohtomo, Y. Ohtsuka, and T. Shimizu, "Uncertainty in management of childhood-onset idiopathic nephrotic syndrome: is the long-term prognosis really favorable?" Pediatric Nephrology, vol. 28, no. 12, pp. 2235-2238, 2013.

[4] I. A. Hauser, E. Schaeffeler, S. Gauer et al., "ABCB1 genotype of the donor but not of the recipient is a major risk factor for cyclosporine-related nephrotoxicity after renal transplantation," Journal of the American Society of Nephrology, vol. 16, no. 5, pp. 1501-1511, 2005.
[5] R. C. Wiggins, "The spectrum of podocytopathies: a unifying view of glomerular diseases," Kidney International, vol. 71, no. 12, pp. 1205-1214, 2007.

[6] Y. Chen, Z. Gong, X. Chen et al., "Tripterygium wilfordii Hook F (a traditional Chinese medicine) for primary nephrotic syndrome," Cochrane Database of Systematic Reviews, vol. 8, Article ID CD008568, 2013.

[7] Y. Chen, Y. Deng, Z. Ni et al., "Efficacy and safety of traditional chinese medicine (Shenqi particle) for patients with idiopathic membranous nephropathy: a multicenter randomized controlled clinical trial," American Journal of Kidney Diseases, vol. 62, no. 6, pp. 1068-1076, 2013.

[8] B. Zhu, Y. Wang, M. Jardine et al., “Tripterygium preparations for the treatment of CKD: a systematic review and metaanalysis," The American Journal of Kidney Diseases, vol. 62, no. 3, pp. 515-530, 2013.

[9] X.-P. Chen, S.-Q. He, X. Zhao, Z.-Y. Huang, and C.-H. Li, "Chinese medicine Extractum trametes robiniophila murr augment tumor necrosis factor related apoptosis-inducing ligand induced apoptosis in human hepatic cancer cell lines," Zhonghua Wai Ke Za Zhi, vol. 43, no. 23, pp. 1524-1527, 2005.

[10] C. Zhu, S. Huang, G. Ding et al., "Protective effects of Huang Qi Huai granules on adriamycin nephrosis in rats," Pediatric Nephrology, vol. 26, no. 6, pp. 905-913, 2011.

[11] Ş. Güçer, B. Talim, E. Aşan et al., "Focal segmental glomerulosclerosis associated with mitochondrial cytopathy: report of two cases with special emphasis on podocytes," Pediatric and Developmental Pathology, vol. 8, no. 6, pp. 710-717, 2005.

[12] W. Wang, Y. Wang, J. Long et al., "Mitochondrial fission triggered by hyperglycemia is mediated by ROCK1 activation in podocytes and endothelial cells," Cell Metabolism, vol. 15, no. 2, pp. 186-200, 2012.

[13] M. Su, A.-R. Dhoopun, Y. Yuan et al., "Mitochondrial dysfunction is an early event in aldosterone-induced podocyte injury," The American Journal of Physiology - Renal Physiology, vol. 305, no. 4, pp. F520-F531, 2013.

[14] Y. Yuan, S. Huang, W. Wang et al., "Activation of peroxisome proliferator-activated receptor- $\gamma$ coactivator $1 \alpha$ ameliorates mitochondrial dysfunction and protects podocytes from aldosterone-induced injury," Kidney International, vol. 82, no. 7, pp. 771-789, 2012.

[15] C. Zhu, X. Xuan, R. Che et al., "Dysfunction of the PGC$1 \alpha$-mitochondria axis confers adriamycin-induced podocyte injury," American Journal of Physiology-Renal Physiology, vol. 306, no. 12, pp. F1410-F1417, 2014.

[16] V. W. Lee and D. C. Harris, "Adriamycin nephropathy: a model of focal segmental glomerulosclerosis," Nephrology, vol. 16, no. 1, pp. 30-38, 2011.

[17] P. Mundel, J. Reiser, and W. Kriz, "Induction of differentiation in cultured rat and human podocytes," Journal of the American Society of Nephrology, vol. 8, no. 5, pp. 697-705, 1997.

[18] S. Huang, A. Zhang, G. Ding, and R. Chen, "Aldosteroneinduced mesangial cell proliferation is mediated by EGF receptor transactivation," The American Journal of Physiology: Renal Physiology, vol. 296, no. 6, pp. F1323-F1333, 2009.

[19] B. M. Acton, A. Jurisicova, I. Jurisica, and R. F. Casper, "Alterations in mitochondrial membrane potential during preimplantation stages of mouse and human embryo development," Molecular Human Reproduction, vol. 10, no. 1, pp. 23-32, 2004.

[20] L. Galluzzi, O. Kepp, C. Trojel-Hansen, and G. Kroemer, "Mitochondrial control of cellular life, stress, and death," Circulation Research, vol. 111, no. 9, pp. 1198-1207, 2012. 
[21] Q. Zhang, K. Itagaki, and C. J. Hauser, "Mitochondrial DNA is released by shock and activates neutrophils via P38 map kinase," Shock, vol. 34, no. 1, pp. 55-59, 2010.

[22] T. Bertani, A. Poggi, R. Pozzoni et al., "Adriamycin-induced nephrotic syndrome in rats. Sequence of pathologic events," Laboratory Investigation, vol. 46, no. 1, pp. 16-23, 1982.

[23] T. S. Lahoti, D. Patel, V. Thekkemadom, R. Beckett, and S. D. Ray, "Doxorubicin-induced in vivo nephrotoxicity involves oxidative stress-mediated multiple pro- and anti-apoptotic signaling pathways," Current Neurovascular Research, vol. 9, no. 4, pp. 282-295, 2012.

[24] R. S. Balaban, S. Nemoto, and T. Finkel, "Mitochondria, oxidants, and aging," Cell, vol. 120, no. 4, pp. 483-495, 2005.

[25] V. Temkin, Q. Huang, H. Liu, H. Osada, and R. M. Pope, "Inhibition of ADP/ATP exchange in receptor-interacting proteinmediated necrosis," Molecular and Cellular Biology, vol. 26, no. 6, pp. 2215-2225, 2006.

[26] T. Kanki and D. J. Klionsky, "The molecular mechanism of mitochondria autophagy in yeast," Molecular Microbiology, vol. 75, no. 4, pp. 795-800, 2010.

[27] P. J. Adhihetty, G. Uguccioni, L. Leick, J. Hidalgo, H. Pilegaard, and D. A. Hood, "The role of PGC-1 $\alpha$ on mitochondrial function and apoptotic susceptibility in muscle," The American Journal of Physiology-Cell Physiology, vol. 297, no. 1, pp. C217-C225, 2009.

[28] Z. $\mathrm{Lu}, \mathrm{X} . \mathrm{Xu}, \mathrm{X}$. Hu et al., "PGC-1 $\alpha$ regulates expression of myocardial mitochondrial antioxidants and myocardial oxidative stress after chronic systolic overload," Antioxidants and Redox Signaling, vol. 13, no. 7, pp. 1011-1022, 2010.

[29] R. R. Thomas, S. M. Kha, R. M. Smigrodzki et al., "RhTFAM treatment stimulates mitochondrial oxidative metabolism and improves memory in aged mice," Aging, vol. 4, no. 9, pp. 620635, 2012.

[30] P. J. Fernandez-Marcos and J. Auwerx, "Regulation of PGC-1 $\alpha$, a nodal regulator of mitochondrial biogenesis," The American Journal of Clinical Nutrition, vol. 93, no. 4, pp. 884S-890S, 2011.

[31] C. Zhu, S. Huang, Y. Yuan et al., "Mitochondrial dysfunction mediates aldosterone-induced podocyte damage: a therapeutic target of PPAR $\gamma$," The American Journal of Pathology, vol. 178, no. 5, pp. 2020-2031, 2011.

[32] S. Tsuruoka, A. Hiwatashi, J. Usui, and K. Yamagata, "The mitochondrial SIRT1-PGC-1 $\alpha$ axis in podocyte injury," Kidney International, vol. 82, no. 7, pp. 735-736, 2012.

[33] H. Matsumura, A. Ashida, K. Hirano, H. Nakakura, and H. Tamai, "Protective effect of radical scavenger edaravone against puromycin nephrosis," Clinical Nephrology, vol. 66, no. 6, pp. 405-410, 2006.

[34] H. S. Lee, J. Y. Jeong, B. C. Kim, Y. S. Kim, Y. Z. Zhang, and H. K. Chung, "Dietary antioxidant inhibits lipoprotein oxidation and renal injury in experimental focal segmental glomerulosclerosis," Kidney International, vol. 51, no. 4, pp. 1151-1159, 1997.

[35] M. Tahzib, R. Frank, B. Gauthier, E. Valderrama, and H. Trachtman, "Vitamin E treatment of focal segmental glomerulosclerosis: results of an open-label study," Pediatric Nephrology, vol. 13, no. 8, pp. 649-652, 1999.

[36] X. Wang, N. Zhang, Q. Huo, and Q. Yang, "Anti-angiogenic and antitumor activities of Huaier aqueous extract," Oncology Reports, vol. 28, no. 4, pp. 1167-1175, 2012.

[37] N. Zhang, X. Kong, S. Yan, C. Yuan, and Q. Yang, "Huaier aqueous extract inhibits proliferation of breast cancer cells by inducing apoptosis," Cancer Science, vol. 101, no. 11, pp. 2375$2383,2010$. 


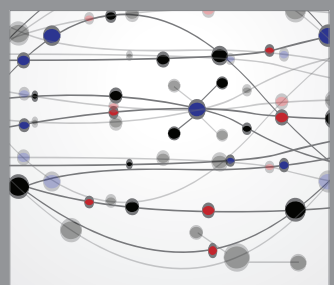

The Scientific World Journal
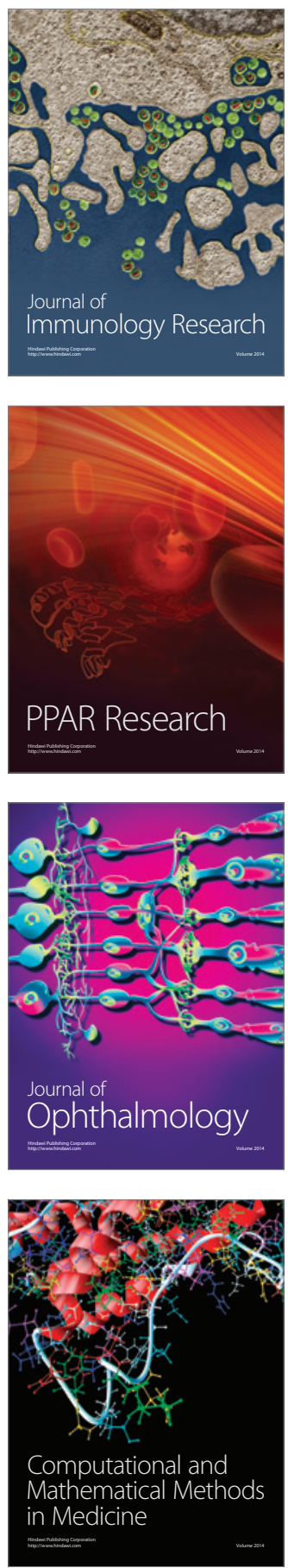

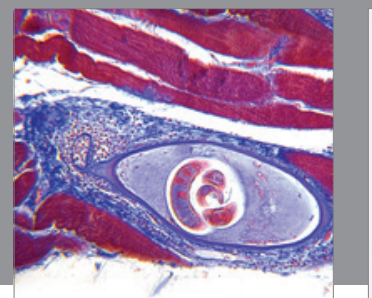

Gastroenterology

Research and Practice
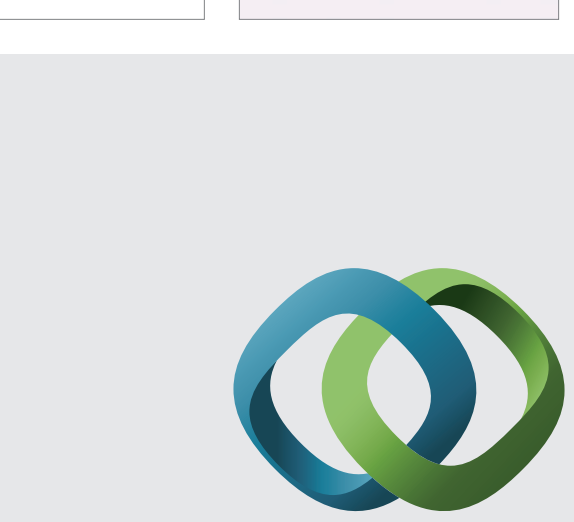

\section{Hindawi}

Submit your manuscripts at

http://www.hindawi.com
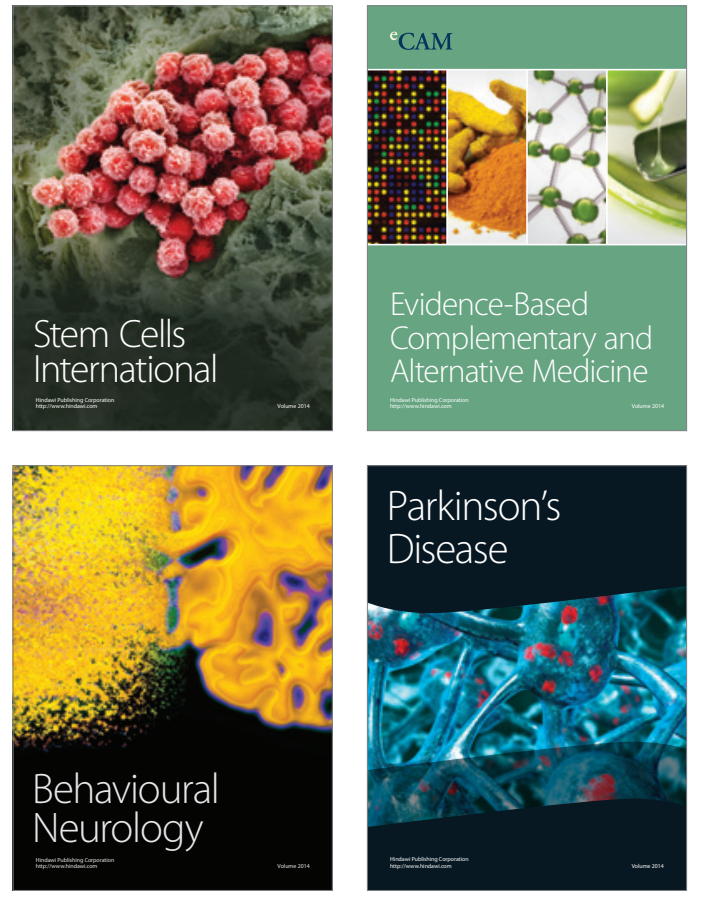
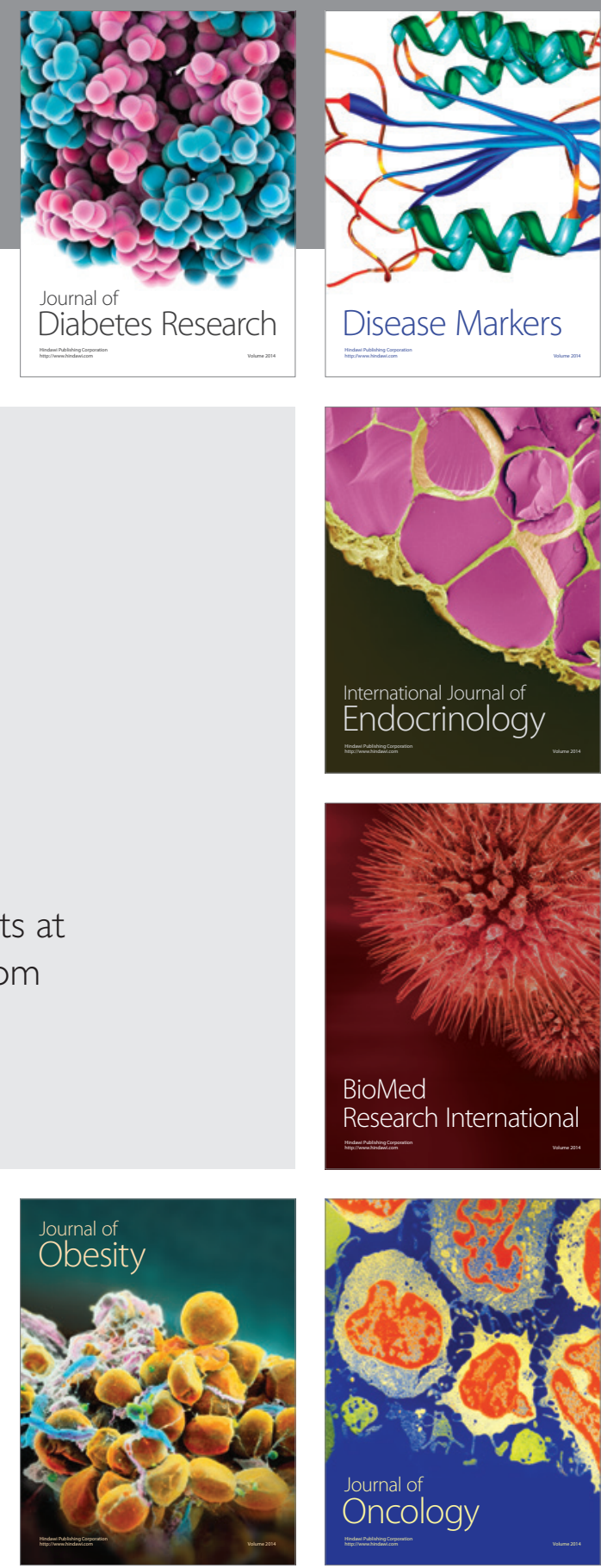

Disease Markers
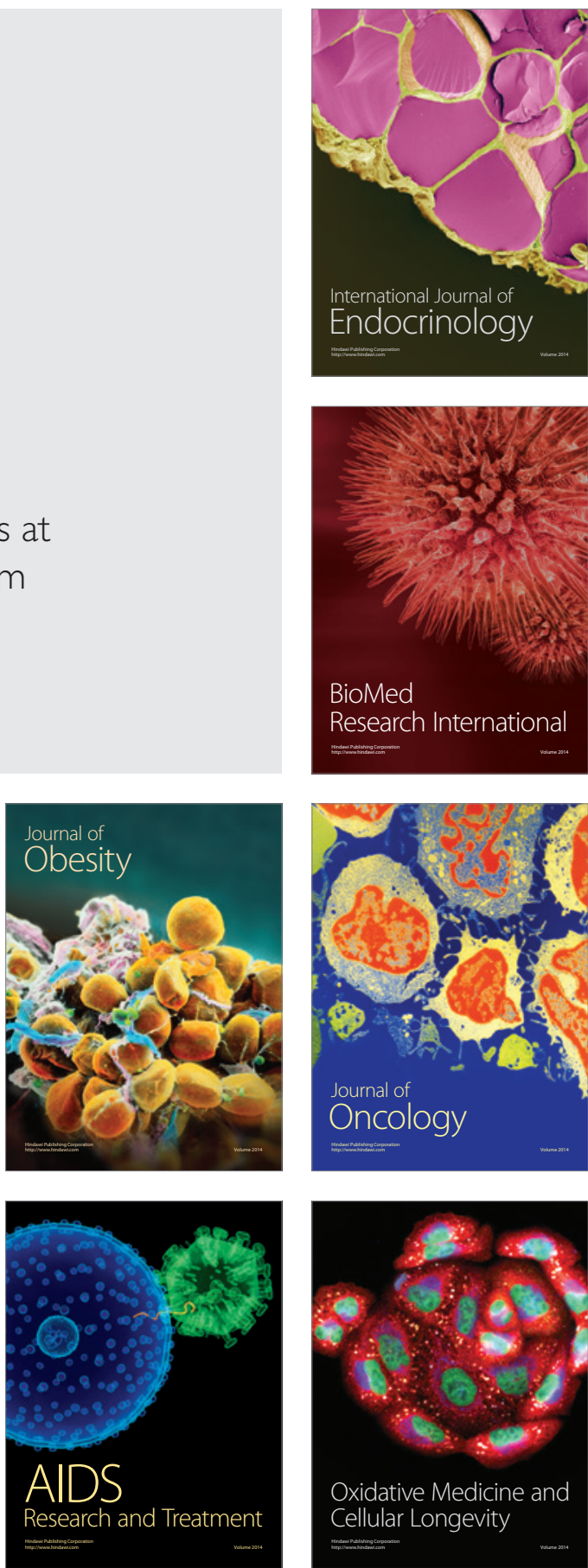